\title{
Deficiencia de hierro y su relación con la función cognitiva en escolares
}

\author{
Ivette C. Rivera*, María Félix Rivera**, Rebeca Rivera***
}

\section{RESUMEN}

Objetivo: En Honduras no se cuenta con estudios de deficiencia de hierro y cognición, por lo que se decidió realizar un estudio con el objetivo de determinar la relación entre la deficiencia de hierro y la capacidad cognitiva en escolares de primer grado de escuelas públicas de Tegucigalpa MDC.

Sujetos y materiales: el estudio es caso control. Se realizo inicialmente un estudio de prevalencia de anemia por deficiencia de hierro y al mismo tiempo un estudio sobre deficiencia de hierro en escuelas públicas de Tegucigalpa en niños de primer grado en el año 2010, de donde se seleccionaron los niños para el presente estudio. Los niños seleccionados se distribuyeron en dos grupos, aparejados de acuerdo a edad y sexo y el nivel socioeconómico, y todos tenían niveles de folatos y cobalamina normales conformados así: Grupo 1: niños con niveles de hemoglobina mayor de $11.4 \mathrm{gr} / \mathrm{dl}$, con ferritina menor de $15 \mu \mathrm{g} / \mathrm{l}$, definidos como "niños casos". Grupo 2: niños con niveles de hemoglobina mayores de $11.4 \mathrm{gr} / \mathrm{dl}$ y ferritina normal, considerados como "niños controles". Para la determinación de la deficiencia de hierro en los niños, se utilizó ferritina. Se les realizaron pruebas psicológicas para determinar la capacidad cognitiva; se evaluó el coeficiente intelectual, atención, memoria y aprendizaje, madurez como ser Raven, Tomal, Bender, D2-Brickenkamp. A los padres de los escolares se les solicitó consentimiento informado y firmado. Se aplicó estadística descriptiva y, para detectar diferencias significativas entre los dos grupos del estudio, la prueba diferencias de promedios ( $t$ de Student) con un valor de p significativo menor a 0,05.

Resultados: la media de edad en el grupo de los niños casos fue de 6.49 años (0.74

\footnotetext{
Universidad Nacional Autónoma de Honduras. Facultad de Ciencias Sociales. Escuela de Psicología. Psicóloga Asistencial del Centro Odontopediátrico y Detección de Otras Patologías (Codopa) de la Región Sanitaria Metropolitana. Secretaría de Salud. prosperoadri@yahoo.com

Universidad Nacional Autónoma de Honduras. Facultad de Medicina. Departamento de Fisiología. Médica Asistencial del Centro Odontopediátrico y Detección de Otras Patologías (Codopa de la Región Sanitaria Metropolitana.Secretaría de Salud.mariafelixrivera@yahoo.com

Universidad Nacional Autónoma de Honduras. Facultad de Ciencias. Escuela de Microbiología.

deliariverac@yahoo.com
} 
1DE) y en los niños casos de 6.27 años (0.45 1DE) las diferencias no fueron estadísticamente significativas $(p=0.26)$. Se encontró que fue menor el puntaje en aprendizaje y memoria en niños con ferritina baja que los que tenían ferritina normal, ambos grupos sin anemia, y las diferencias fueron estadísticamente significativas $(p=0.02)$ y no en los puntajes de atención ya que no se encontraron diferencias significativas $(p=0.81)$ entre los grupos.

Conclusiones: como hemos visto, la mayoría de los estudios realizados en escolares con deficiencia de hierro coincide en vislumbrar un deterioro de dicha capacidad haciendo evidente que los escolares con déficit de hierro sin anemia tendrán problemas en el aprendizaje y por ende va a repercutir en el aprovechamiento escolar, por lo que se deben de tomar medidas necesarias y urgentes para la solución de este problema totalmente prevenible.

\section{Palabras claves: Hierro, cognición, escolares}

\section{ABSTRACT}

Objective: In Honduras there are no studies of iron deficiency and cognition therefore decided to conduct a study as to determine the relationship between iron deficiency and cognitive ability in first-grade public school in Tegucigalpa MDC.

Subjects and materials: case-control study. We performed an initial study of the prevalence of iron deficiency anemia and iron deficiency in public schools in Tegucigalpa first graders in 2010, where children were selected for this study. The children selected were divided into two groups paired according to age, sex and socioeconomic levels and all had normal folate and cobalamin formed as follows: Group 1: children with hemoglobin levels greater than $11.4 \mathrm{~g} / \mathrm{dl}$, ferritin below $15 \mathrm{ug}$ I I defined as "children cases." Group 2: Children with hemoglobin levels greater than $11.4 \mathrm{~g} / \mathrm{dl}$ and ferritin considered normal" control children". For the determination of iron deficiency in children, we used ferritina. Psychological testing was done to tests to determine cognitive psychological and assessed IQ, attention, memory and learning, maturity, such as Raven, Tomal, Bender, D2-Brickenkamp. Parents of schoolchildren were asked and signed informed consent. Descriptive statistics were applied to detect significant differences between the two studies groups, the test mean differences (t test) with a significant $p$ value less than 0.05 .

Results: The mean age in the group of children was 6.49 years cases $(0.741 \mathrm{SD})$ and children in cases of 6.27 years $(0.451 \mathrm{SD})$ the differences were not statistically significant $(p=0.26)$. was found to lower the score in learning and memory in 
children with low ferritin levels than those with normal ferritin without anemia and both groups were statistically significant differences $(p=0.02)$ and not so in care scores no differences significant $(p=0.81)$ between groups.

Conclusions: As we have seen most of the studies in iron-deficient school agree envision a deterioration in that school becoming clear that iron deficiency without anemia have learning problems and thus will affect the use school so they must take urgent and necessary measures to solve this problem entirely preventable.

Keywords: Iron, cognition, school children 


\section{INTRODUCCIÓN}

La deficiencia de hierro afecta a más de 2,000 millones de personas en todo el mundo, aproximadamente 77 millones viven en América Latina, los más afectados son las embarazadas y los niños menores de 5 años pero también escolares aunque en menor grado pero trascendente por su relación con el bajo rendimiento escolar ${ }^{1,2}$. Existen muchos estudios en los últimos 30 años que documentan los efectos que la anemia por deficiencia de hierro provoca en el desarrollo mental de los niños e infantes ${ }^{3-9}$. Sin embargo se ha estado en la búsqueda de las alteraciones que provoca la deficiencia de hierro sin anemia. Por mucho tiempo se han realizado estudios buscando una relación entre los efectos que pueden tener este déficit nutricional sobre el cerebro, principalmente durante la gestación y los primeros años de vida, o sea en la fase de crecimiento rápido neuronal, por lo que la mayoría de los estudios se han concentrado en menores de dos años de edad ${ }^{11}$.

Existe un consenso científico sobre el papel que juegan las deficiencias de micronutrientes en el desarrollo cognitivo y motor de los niños, en la conducta y en el logro escolar. Entre tales micronutrientes se encuentra el hierro. El cerebro presenta áreas que son ricas en hierro y experimentalmente se ha observado que son sensibles a la depleción de hierro ${ }^{12}$. El hierro es un componente clave de muchas enzimas involucradas en las reacciones esenciales de óxido reducción y en la síntesis y catabolismo de neurotransmisores. El hierro es cofactor de la ribonucleasa reductasa, enzima que cataliza la etapa limitante de la síntesis de ADN y también es requerido para la apropiada mielinización de la médula espinal y cerebelo ${ }^{13}$.

La revisión realizada por Grantham señala que la mayoría de los estudios de correlación han encontrado asociación entre la anemia por deficiencia de hierro y el pobre desarrollo cognitivo, motor y problemas conductuales. Los estudios longitudinales indicaban que los niños que experimentaron anemia durante su vida temprana continúan demostrando bajo rendimiento académico durante sus años escolares, aun después de que la anemia ha sido tratada ${ }^{14}$. Las consecuencias de la deficiencia de hierro en la función neuronal no son restrictivos a la infancia o a preescolares ya que varios estudios sugieren que el aprendizaje está afectado tanto en escolares, adolescentes e incluso adultos depletados de hierro ${ }^{15-19}$. Una insuficiente disponibilidad de hierro en un período de alta incorporación de éste en el tejido cerebral, que coincide con el período de mielinización del tejido nervioso, puede proveer una base fisiológica para explicar los efectos conductuales observados cuando hay deficiencias del micronutriente. De la misma manera, la deficiencia de hierro afecta la regulación y la conducción de neurotransmisores 
como la serotonina, la dopamina y GABA. La alteración de los receptores y transportadores de dopamina, compromete en los infantes las respuestas afectivas y el funcionamiento cognoscitivo, y de los receptores GABA, la coordinación de patrones de movimiento y memoria. La importancia consiste que cuando ocurre un déficit de hierro cerebral en etapas tempranas, los daños ocurridos persisten en la etapa adulta, más allá de la recuperación de la anemia durante los primeros meses de vida.

Estas alteraciones cerebrales se reflejan a largo plazo en un retraso del desarrollo mental y físico de los niños que han tenido anemia, y como consecuencia un menor desempeño escolar, con altos niveles de repetición de grados y deserción de la escuela primaria en comunidades económicamente pobres. La persistencia de estas alteraciones en la edad adulta, después de corregir la deficiencia, se evaluó recientemente, e indican que la cantidad de receptores de dopamina D2R es más baja en la sustancia nigra, mientras que los de serotonina se encuentran en menor densidad en los núcleos laterales y reticulares del tálamo. El déficit en el hipocampo y la corteza se relaciona con deficiencias en el aprendizaje espacial. La alteración de los receptores de dopamina, compromete en los infantes las respuestas afectivas, y de esa forma su relación con el ambiente y el funcionamiento cognitivo ${ }^{20}$. En Honduras no se cuenta con estudios de deficiencia de hierro y cognición, por lo que este estudio tiene como objetivo determinar la relación entre la deficiencia de hierro y la capacidad cognitiva en escolares de primer grado de escuelas públicas de Tegucigalpa MDC.

\section{SUJETOSYMÉTODOS}

El estudio es tipo caso control. Se realizó inicialmente un estudio de prevalencia de anemia por deficiencia de hierro y deficiencia de hierro en escuelas públicas de Tegucigalpa en niños de primer grado en el año 2010, de donde se seleccionaron los niños para el presente estudio. Los niños ya seleccionados se distribuyeron en dos grupos aparejados de acuerdo a edad, sexo y el nivel socioeconómico y todos tenían niveles de folatos y cobalamina normales conformados así: Grupo 1: niños con niveles de hemoglobina mayor de $11.4 \mathrm{gr} / \mathrm{dl}$, con ferritina menor de $15 \mu \mathrm{g} / \mathrm{l}$ definidos como "niños casos". Grupo 2: niños con niveles de hemoglobina mayores de $11.4 \mathrm{gr} / \mathrm{dl}$ y ferritina normal, considerados como "niños controles".

Laboratorio: Se les habían extraído $5 \mathrm{ml}$ de sangre mediante punción venosa cubital con jeringas y agujas en horario matutino, $2 \mathrm{ml}$ se depositaron en tubos con EDTA para efectuar los hemogramas, utilizando un contador hematológico automatizado ABX Pentra 120; los $3 \mathrm{ml}$ restantes se centrifugaron para obtener 
suero, el cual se congeló en dos alicuotas para efectuar un estudio de niveles de ferritina, ácido fólico y vitamina B12; los cuales se determinaron mediante el enzimoinmunoanálisis de micropartículas utilizando el sistema automatizado AxSYM. Las mediciones fueron realizadas por personal altamente calificado en el laboratorio de hematología de la Carrera de Microbiología de la Universidad Nacional Autónoma de Honduras. Los niveles de hemoglobina mayores de $11.4 \mathrm{~g} / \mathrm{dl}$ se consideraron como anémicos para la edad de 5 a 12 años ${ }^{21}$.

En cuanto a los parámetros nutricionales bioquímicos para la determinación de la deficiencia de hierro en los niños, se utilizó ferritina sérica considerando que la disminución de la ferritina implica que los depósitos de hierro están disminuidos, siendo la principal proteína que almacena el hierro, y es directamente proporcional a la cantidad de hierro almacenado. Se consideró niveles bajos de ferritina menor de $15 \mu \mathrm{g} / \mathrm{l}$ según las normas internacionales emitidas por la OPS y OMS. También se consideró los niveles de cobalamina y folatos y fueron definidos según OPSOMS considerando como niveles bajos de cobalamina menores a $203 \mathrm{pg} / \mathrm{ml}$ y de folatos menores a $4 \mathrm{ng} / \mathrm{ml}^{22,23}$.

Estado nutricional: A todos los niños se les tomó talla y peso para clasificar el crecimiento según los parámetros propuestos por el Instituto de Nutrición de Centroamérica y Panamá (INCAP) para niños mayores de cinco años aceptados por el Centro Nacional de Estadística para la Salud de Estados Unidos de América (NCHS) y la Organización Mundial de la Salud (OMS) donde estima: retardo moderado del crecimiento, talla entre -2 y -2.9 desviaciones estándar (DE) y retardo severo, igual o menos de -3DE ${ }^{24}$. El nivel socioeconómico: Se estratificó según el Índice de Graffar modificado que incluye índice de vivienda (materiales de construcción, disposición de excretas y agua), escolaridad y ocupación del jefe de $\operatorname{hogar}^{25}$.

Estudio psicológico: Se le realizaron pruebas psicológicas para determinar capacidad cognitiva; se evaluó coeficiente intelectual, atención, memoria y aprendizaje y madurez viso motora. Para medir la inteligencia se utilizó el test de Raven ya que es un test que mide la capacidad intelectual comparando formas y razonando analogías independientemente de la cultura, de los conocimientos adquiridos, de la habilidad verbal y manual, y de acuerdo al puntaje total obtenido se estableció el diagnóstico según los percentiles proporcionados por Raven. Se utilizó el D2-Brickenkamp como test para medir atención selectiva y concentración y Tomal como test de memoria y aprendizaje. Para madurez y percepción visomotora se utilizó el test de Bender. Para la aplicación de los test se utilizó personal calificado en administración de pruebas psicológicas. 
Consideraciones éticas: A los padres de los escolares estudiados se les solicitó consentimiento informado y firmado.

Criterios de exclusión: Fueron excluidos de los estudios niños sin autorización firmada por los padres, niños con $\mathrm{Cl}$ bajo proporcionado por Raven, con antecedentes de convulsiones, traumatismo encéfalocraneano, prematuros, bajo peso al nacer, antecedentes de hipoxia neonatal, desnutrición crónica, aguda y global.

Análisis: Se aplicó estadística descriptiva determinando distribución de frecuencias, medidas de tendencia central como la media y medidas de dispersión como la Desviación Estándar (DE). Para detectar diferencias significativas en los dos grupos de estudio entre las variables, se utilizó la prueba diferencias de promedios (t de Student) con un valor de p significativo menor a 0,05.

\section{RESULTADOS}

El estudio contó con un total de 45 niños distribuidos de la siguiente manera: 15 en el grupo de niños con ferritina baja sin anemia, o sea "casos" y 30 en el grupo "control". La media de edad fue de los niños con ferritina baja de $6.4 \pm 0.74$ años 1Desviación Estándar (1DE) y de 6.2 años \pm 0.45 en los controles y las diferencias no fueron significativas $p=0.26$. En relación a la madurez visomotora medida por el test de Bender, así como en el test D2 que mide atención, las diferencias no fueron estadísticamente significativas al igual que en concentración y variabilidad, como se puede observar en el cuadro No. 1.

Cuadro No. 1. Comparación de la madurez visomotora, la concentración y atención entre los niños casos y niños controles.

\begin{tabular}{|l|c|c|c|c|}
\hline \multicolumn{1}{|c|}{ Variable } & Niños casos & Niños controles & $\mathbf{t}$ & $\mathbf{p}$ \\
\hline Bender madurez & 6.16 & 2.35 & 1.22 & 0.22 \\
\hline Visomotora & $(3.01)$ & $(2.73)$ & & \\
\hline D2 & 171.07 & 169.6 & 0.08 & 0.93 \\
& $(60.49)$ & $(49.12)$ & & \\
\hline Concentración & 48.57 & 62.92 & 1.27 & 0.21 \\
& $(25.71)$ & $(37.58)$ & & \\
\hline Variabilidad & 20.28 & 12.42 & 1.30 & 0.20 \\
& $(30.45)$ & $(4.75)$ & & \\
\hline
\end{tabular}

Entre paréntesis se presenta la desviación estándar, $p<0.05$ es significativo. 
Al evaluar memoria y aprendizaje a través del test Tomal en el subtest verbal, en el ítem de recuerdo selectivo de palabras, se observó un menor puntaje en los "niños casos" que en los "niños controles", así mismo en el recuerdo de objetos y fue estadísticamente significativo ( $p=0.003$ y 0.02 respectivamente) y también en la subescala de recuerdo demorado el puntaje fue menor en el grupo de los niños casos que en los controles y las diferencias fueron estadísticamente significativas $(p=0.03)$ y por supuesto esto repercute en el índice de memoria verbal, donde también los puntajes fueron más bajos en el grupo caso que en el control y las diferencias fueron estadísticamente significativas $(p=0.02)$. En el resto de las escalas, las diferencias no fueron estadísticamente significativas como se puede observar en el cuadro No.2.

Cuadro No. 2. Comparación del test psicológico Tomal de memoria y aprendizaje entre los niños casos y los controles.

\begin{tabular}{|l|c|c|c|c|}
\hline \multicolumn{1}{c|}{ Items } & $\begin{array}{c}\text { Niños } \\
\text { casos }\end{array}$ & $\begin{array}{c}\text { Niños } \\
\text { controles }\end{array}$ & $\mathbf{t}$ & $\mathbf{p}$ \\
\hline Subtest verbal & & & & \\
\hline Memoria de historias & $7.84(3.05)$ & $8.82(2.34)$ & 1.12 & 0.26 \\
\hline Recuerdo selectivo de palabras & $7.61(2.93)$ & $11.18(3.50)$ & 3.16 & 0.003 \\
\hline Recuerdo de objetos & $8.15(1.77)$ & $10.03(2.48)$ & 2.43 & 0.02 \\
\hline Dígito directo & $7.84(1.67)$ & $8.41(2.41)$ & 0.76 & 0.44 \\
\hline Recuerdo de pares & $8.92(2.81)$ & $9.66(3.10)$ & 0.71 & 0.47 \\
\hline Letras directo & $8.76(1.58)$ & $8.86(1.54)$ & 0.18 & 0.85 \\
\hline Dígitos inverso & $8.53(2.25)$ & $8.26(2.76)$ & 0.30 & 0.76 \\
\hline Letras inverso & $8.46(2.90)$ & $8.13(2.00)$ & 0.40 & 0.68 \\
\hline Subtest no verbales & & & & \\
\hline Memoria de caras & $9.84(2.91)$ & $10.28(2.73)$ & 0.46 & 0.64 \\
\hline Recuerdo selectivo visual & $8.30(2.78)$ & $8.48(3.16)$ & 0.16 & 0.86 \\
\hline Memoria visual abstracta & $9.23(3.08)$ & $10.25(3.42)$ & 0.89 & 0.37 \\
\hline Memoria secuencial visual & $9.84(3.05)$ & $8.54(3.51)$ & 1.12 & 0.26 \\
\hline Memoria de lugares & $8.69(1.79)$ & $9.26(2.02)$ & 0.84 & 0.40 \\
\hline Invitación manual & $10.15(1.46)$ & $9.26(1.68)$ & 1.58 & 0.11 \\
\hline Subescalas para recuerdo demorado & & & & \\
\hline Recuerdo demorado de memoria de historias & $7.66(2.87)$ & $9.4(2.21)$ & 2.02 & 0.05 \\
\hline Recuerdo demorado de memorias de caras & $12.38(1.38)$ & $11.6(1.76)$ & 1.5 & 0.13 \\
\hline Recuerdo demorado selectivo de palabras & $10.58(0.99)$ & $11.40(1.11)$ & 2.15 & 0.03 \\
\hline Recuerdo demorado recuerdo selectivo visual & $9.72(1.61)$ & $9.16(1.51)$ & 1.01 & 0.31 \\
\hline Totales & & & & \\
\hline Índice de memoria verbal & $86.0(12.86)$ & $95.41(11.26)$ & 2.31 & 0.02 \\
\hline Índice de memoria no verbal & $92.07(14.92)$ & $93.47(11.37)$ & 0.31 & 0.75 \\
\hline Índice de memoria compuesta & $87.46(11.68)$ & $94.52(10.90)$ & 1.81 & 0.07 \\
\hline Índice de recuerdo demorado & $96.54(9.55)$ & $101.11(8.21)$ & 1.47 & 0.14 \\
\hline
\end{tabular}

Entre paréntesis se presenta la desviación estándar, $p<0.05$ es significativo. 
En resumen se encontró que fue menor el puntaje en aprendizaje y memoria en niños con ferritina baja que los que tenían ferritina normal, ambos grupos sin anemia, y las diferencias fueron estadísticamente significativas $(p=0.02)$ y no así en los puntajes de atención, donde no se encontraron diferencias significativas $(p=0.81)$ como se puede observar en el cuadro No. 3

Cuadro No. 3. Resumen de los puntajes obtenidos en relación a aprendizaje y atención comparativo entre niños casos y controles.

\begin{tabular}{|l|c|c|c|c|}
\hline \multicolumn{1}{|c|}{ Variable } & Niños casos & Niños controles & $\mathbf{t}$ & $\mathbf{p}$ \\
\hline Aprendizaje & $82.92(12.82)$ & $93.77(14.66)$ & 2.27 & 0.02 \\
\hline Atención & $88.69810 .97)$ & $88.87(9.71)$ & 0.23 & 0.81 \\
\hline
\end{tabular}

Entre paréntesis se presenta la desviación estándar, $p<0.05$ es significativo.

\section{DISCUSIÓN}

Uno de los problemas que enfrenta este estudio es el no contar con una historia o datos anteriores de anemia por deficiencia de hierro o deficiencia de hierro sino que es un corte transversal, porque los daños más severos se presentan en la primeras etapas de la vida, sin embargo es un aporte de parte nuestra para la ciencia en cuanto a la relación que existe entre deficiencia de hierro y función cognitiva en una población escolar. Se utilizó como parámetro de deficiencia de hierro el nivel de ferritina únicamente. Cuando existen problemas inflamatorios la ferritina aumenta falsamente, pero en este estudio solo se tomó escolares con ferritina por debajo de $15 \mu \mathrm{g} / \mathrm{dl}$, por lo que es irrelevante.

En nuestra investigación se encontró que los niños con deficiencia de hierro sin anemia obtenían menores puntajes en test psicológicos de memoria, en recuerdo selectivo de palabras y recuerdo de objetos, así mismo en el índice de memoria verbal, pero no se encontró diferencias en cuanto a atención y madurez visomotora.

Montilva y Padród, en Venezuela, estudió la relación entre el status de hierro en escolares midiendo también atención-concentración, memoria, razonamiento y discriminación visual. El promedio de puntuación en prueba de dígitos y aritmética, en niños con status de hierro normal fue de 6,57 y 8,78 respectivamente, mientras 
que en deficientes de hierro fue menor de 5,25 y $7,10(p<0,03)$. En las pruebas de claves y figuras incompletas, que además miden la coordinación visomotora y organización visual, no se encontraron diferencias significativas al comparar los grupos al igual que en nuestro estudio ${ }^{26}$.

Un estudio realizado en Estados Unidos en escolares encontró que los promedios de matemáticas que tienen que ver con memoria y repetición de dígitos eran más bajos en niños con deficiencia de hierro, comparados con los que tenían un estado de hierro normal ${ }^{27}$. También una investigación realizada por Ortega y Colaboradores "en escolares" encontraró los mismos hallazgos que nuestro estudio, o sea una afectación de la memoria y concluyó que los procesos de atención alterados se pueden ver en niños con anemia y posiblemente los de memoria estén más asociados con el déficit de hierro por ser más sensibles a la disminución de este mineral, con lo que estamos de acuerdo ${ }^{28}$.

Los hallazgos en estos estudios apoyan el concepto que no es necesario que el deterioro del estado del hierro avance hasta su última etapa, como ser la anemia, para que la función cognitiva en los escolares sea vulnerable y sufra cambios al igual que lo encontrado en el presente estudio.

Por otra parte, si bien es cierto que en nuestro estudio no se detectaron déficits en capacidad de atención, ni madurez visomotoras en los niños con déficit de hierro, una investigación en escolares adolescentes, clasificados como deficientes en hierro, mostró algunos problemas funcionales específicos en las áreas de atención de organización visual-perceptual, aunque también en memoria visual-espacial y memoria incidental $^{29}$.

Las formas de medir la capacidad cognitiva varían de un estudio a otro, pero como hemos visto, la mayoría de los estudios realizados en escolares con deficiencia de hierro coincide en vislumbrar un deterioro de dicha capacidad haciendo evidente que los escolares con déficit de hierro sin anemia tendrán problemas en el aprendizaje y por ende va a repercutir en el aprovechamiento escolar, por lo que se deben de tomar medidas necesarias y urgentes para la solución de este problema totalmente prevenible.

\section{BIBLIOGRAFÍA}

1. Naciones Unidas. Sub-Comité de Nutrición de las Naciones Unidas. Comités de Coordinación Administrativa. Estado de la situación mundial en nutrición. Ginebra; 2000. 
2. Erikson KM, Jones $\mathrm{BC}$, Beard JL. Iron deficiency alters dopamine transporter functioning in rat stratium. J Nutr 2000; 130:2831-7

3. Beard JL. Iron biology in immune function, muscle metabolism, and neuronal functioning. J Nutr 2001;131:568S-580S

4. Beard JL, Connor JR, Jones BC. Iron in the Brain. Nutr Rev. 1993;51:157-70

5. Beard JL, Erikson K. Jones BC. Neurobehavioral analysis of development iron deficiency in rats. Behav Brain Res 2002; 134:517-24

6. Sungthong R, Mo-Suwan L, Chongsuvivatwong V. Effects of hemoglobin and serum ferritin on cognitive function in school children. Asia Pac J Clin Nutr 2002;11:117-122

7. Sen A, Kannani Sj. Deleterious functional impact of anemia on young adolescent school girls. Indian Pediatric 2006;43:219-226

8. Halterm JS, Kaczorowski JM, Aligne CA, Auinger P, Szilagyi PG. Iron deficiency and cognitive achievement among school-aged children and adolescents in the United States. Pediatrics 2001;107:1381-1386

9. Motilva M, Padrón AG. Deficiencia de hierro y algunas funciones cognitivas en escolares. An Venez Nutr 2000;13:196-201.

10. Pollit $E$. Timing and vulnerability in research on malnutrition and cognition. Nutr Rev 1996;54:S49-S55

11. Bryan J, Osendarp S, Hughes D, Calvaresi E, Baghurst K, Van Klinken JW. Nutrients for cognitive development in school-aged children. Nutr Rev 2004;62:295-306

12. Beard J. Iron biology in immune function, muscle metabolism and neural function. J. Nutr 2001;131:568S-579S

13. Beard J. Iron deficiency alters brain development and functioning. J Nutr 2003;133(5Suppl1):1468S-1472

14. Grantham-McGregor S, Ani C. A review of studies on the effect of iron deficiency on cognitive development in children. J Nutr 2001;131:649S-658S

15. Groneer JA, Holtzman NA, Charney E, Mellitts E. A randomized triat of oral iron on test of short term memory and attention span in young pregnant women. $J$ Adolesc Health Care 1986; 7:44-48

16. Kashyap P, Gopaldas T. Impact of hematinic supplementation on cognitive function in underprivileged school girls (8-15 Yrs of age) Nutr Res1987;7:111726

17. Pollit $E$, Hathirat $P$, kotchabhakdi NJ, Missell $L$, Valyasevi $A$. iron deficiency and educational achievement in Thailand. Am J Clin Nutr 1983;50:687-97

18. Seshadri S, Gopaldas T. Impact of iron supplementation on cognitive functions in preschool and school aged children: the Indian experience. Am J Clin Nutr. 1989;50:675-86

19. Soemantri AG, Pollit E, Kim I. iron deficiency anemia and educational 
achievement. Am J Clin Nutr 42:1221-28

20. Beard JL, Felt B, Schallert T, Burhans M, Connor JR, Georgieff MK. Moderate iron deficiency in infancy: Biology and behavior in young rats. Behav Brain Res 2006; 170: 224-232.

21. World Health Organization. Iron Deficiency Anemia. Assessment, Prevention and Control. Aguide for programme managers.2001

22. Assessing the iron status of populations: report of a joint World Health Organization/Centers for Disease Control and Prevention Technical consultation on the assessment of iron at the population level, $2^{\text {nd }} \mathrm{ed}$, Geneva, World Health Organization, 2007.

(http:www.who.int/nutrition/publications/micronutrients/anaemia_iron_ deficiency/9789241596107.pdf. consultado el 10 de marzo 2012).

23. World Health Organization. Conclusions of a WHO Technical Consultation on folate and vitamin B12 deficiencies. Food and Nutrition Bulletin 2008; 29 (supplement), S238-S246

24. Organización Mundial de la Salud. Anemias nutricionales. Serie Informes Técnicos No 405. Ginebra: OMS;1968. pp.30.

25. Álvarez M de la L, Muzzo S, Ivanovic D. Escala para la medición del Nivel socioeconómico en el área de salud. Rev Med Chile 1985;113:243-249

26. Montilva M, Padród AG. Deficiencia de hierro y algunas funciones cognitivas en escolares. An Venez nutr 200; 13:196-201

27. Halterman JS, Kaczorowski JM, Aligne CA, Auinger P, Szilagyi PG. Iron deficiency and cognitive achievement among school-aged children and adolescent in the United States. Pediatrics 2001;107:1381-1386

28. Ortega RM, Gonzales Fernández M, Paz L. Andrés P, Jiménez LM, Jiménez MJ et al. Influence of iron status on attention and intelectual performance of a population of spanish adolescent. Arch Latinoam nutr 1993;43(1):6-11

29. Lozoff B, Jiménez E, Hagen J, Mollen E, Wolf AW. Poorer behavioral and developmental outcome more than 10 years after treatment for iron deficiency in infancy. Pediatrics 2000; 105: E51. 